\title{
AN IN SITU CRYSTAL GROWTH OF METAL ORGANIC FRAMEWORKS-5 ON ELECTROSPUN PVA NANOFIBERS
}

\author{
Hanen Talmoudi ${ }^{1}$, Nabyl Khenoussi ${ }^{3}$, Dominique Adolphe ${ }^{3}$, Ayoub Haj Said $^{1,2}$, Laurence Schacher ${ }^{3}$
}

'Laboratory for Advanced Materials and Interfaces, University of Monastir, Faculty of Sciences of Monastir, environmental Boulevard 5000 Monastir, Tunisia

${ }^{2}$ Research Center for Microelectronics and Nanotechnologies. Technopole Sousse, 4000, Sahloul Sousse, Tunisia

${ }^{3}$ Laboratory of Textile Physics and Mechanics EA 4365, University of Haute Alsace, 11 rue Alfred Werner 68093 Mulhouse Cedex, France

\section{Abstract:}

In this study, a simple, general and straightforward method for growing metal-organic frameworks (MOFs) crystals directly on nanofibers is presented. A chelating polymer was first blent with metal cation and then electrospun. The obtained nanofibers were immersed in a linker solution. Metal cations were released and the metal-organic frameworks crystals were grown on the fibers' surface. In this work, this method was tested with polyvinyl alcohol as chelating polymer, $\mathrm{Zn}^{2+}$ as metal cation and Terephthalic acid as linker. The pair cation/linker corresponds to the MOF-5. The latter is a robust metal organic framework formed from $\mathrm{Zn}_{4} \mathrm{O}$ nodes with 1,4-benzodicarboxylic acid struts between the nodes. SEM images revealed that the MOF-5 nanocrystals have grown along the PVA/Zn ${ }^{2+}$ nanofibers that served as the crystals' growth template by providing the $\mathrm{Zn}^{2+}$ ions. This result was also confirmed by infrared spectroscopy, which indicates the presence of characteristic bands of MOF-5 in the modified nanofibers spectrum. Moreover, the X-ray diffraction showed that MOF-5 material was well crystallized on the nanofibers surface according to a cubic symmetry with a space group Fm-3m and a lattice constant $a=25.8849 \AA$.

\section{Keywords:}

Electrospinning, functional textile, metal organic frameworks, hierarchical structures, MOF-5, PVA

\section{Introduction}

The elaboration of nanofibers has attracted much attention in the past few decades [1,5]. Indeed, nanofibers present many properties such as high surface area-to-volume, pore size within a nano-range, high porosity and flexibility for chemical and physical functionalization [2]. Therefore, these very fascinating properties of nanofibers make them attractive and applicable in several areas such as biotechnology,[6] membranes and filters [7], electroanalysis [8] and so on. In this frame, electrospinning is a simple and versatile technique for producing hierarchical structures and multifunctional nanofibers from various polymers, polymer blends and composites [3-11]. A conventional electrospinning setup consists of a highvoltage electrical source, an electrospinning-jet syringe and a conducting collector [12]. During electrospinning, an external electric field is applied between an electrode contacting with the spinning solution and a grounded collector. The resulting electrostatic repulsion and Coulomb force are the driving forces of drawing the solution droplets from the syringe needle. When the applied electric field exceeds a critical value, the syringe needle will jet out the charged stream and, after the solvent evaporates, nonwoven membranes made of nano- to submicron-sized fibers are obtained on the collector surface.

Recently, electrospinning has been used to build hierarchical structures of different functional material[1]; particularly, of metal-organic frameworks (MOFs) [13-18]. In fact, MOFs are a novel class of crystalline hybrid mesoporous materials resulting from the self-assemblies of metal ions/clusters with polydentate organic linkers [19]. They have drawn considerable interest due to their distinct features including high porosity, large surface area, flexible skeleton and their potential applications in gas storage and separation, catalysis, drug delivery systems, energy storage and sensors elaboration [19]. Note that MOFs were generally prepared as bulk materials by solvothermal syntheses. Consequently, many research endeavors have been focused on structuring MOFs at the mesoscopic/macroscopic scale and on the elaboration of MOFs-coated surfaces and membranes [20-23]. In this frame, electrospinning was used for the development of MOF-functionalized textiles using different methodologies [13-18]. Formerly, polymer nanofibers filled with MOFs were obtained by electrospinning the polymer solution containing dispersed MOFs particles [13-15]. Unfortunately, the performance of MOFs was inevitably decreased because of the additional diffusion barrier created around the MOF particles embedded in the electrospun polymer nanofibers. Thus, more recently, many attempts to grow MOF crystals on polymer nanofibers were described. This methodology involved long and complicated multi-step operations [16-18]. At first, nanofibers were chemically modified by introducing functional groups into the polymer structure. These groups enable the metal cations' entrapment onto the nanofibers surface. After a secondary growth step, crystals grow along the fibers forming MOFs sheath.

In this work, we describe a simple and straightforward methodology based on the in situ conversion of cation-loaded nanofibers to MOFs hierarchical structure. Accordingly, Poly (vinyl alcohol) PVA $/ \mathrm{Zn}^{2+}$ salt solution was electrospun. The choice of PVA was motivated by its capability to form homogenous solution with metallic salts due to its chelating 
character. Moreover, PVA is a non-toxic, biocompatible and biodegradable synthetic polymer. The growth of MOF crystals was tested by the immersion of electrospun nanofibers in a linker solution.

Fourier transform infrared spectroscopy (FT-IR), powder X-ray diffraction (DRX) and scanning electron microscopy (SEM) were used to characterize the different materials prepared in this study.

\section{Experimental}

\subsection{Materials}

Poly (vinyl alcohol) (PVA, Mw= $93400 \mathrm{~g} / \mathrm{mol}, \quad 99-100 \%$ hydrolyzed), zinc nitrate hexahydrate $\left(\mathrm{Zn}\left(\mathrm{NO}_{3}\right)_{2} .6 \mathrm{H}_{2} \mathrm{O}\right)$, 1,4-benzene dicarboxylic acid (commonly known as terephthalic acid, $\mathrm{H}_{2} \mathrm{BDC}$ ), triethylamine (TEA), were provided by Aldrich. All solvents and chemicals are of reagent quality and were used without further purification.

\subsection{Methods}

\subsubsection{Synthesis of PVA/Zn ${ }^{2+}$ solution}

The solution was synthesized with $10 \mathrm{wt} \%$ of Poly (vinyl alcohol) (PVA, $M_{w}=93400 \mathrm{~g} / \mathrm{mol}$ ) in deionized water by vigorous stirring at $90^{\circ} \mathrm{C}$. Afterwards, $2 \mathrm{~g}$ of zinc nitrate hexahydrate $\left(\mathrm{Zn}\left(\mathrm{NO}_{3}\right)_{2}\right.$. $6 \mathrm{H}_{2} \mathrm{O}$ ) were added into the previously prepared PVA solution.

\subsubsection{Electrospinning of $\mathrm{PVA} / \mathrm{Zn}^{2+}$ nanofibers}

The solution of $\mathrm{PVA} / \mathrm{Zn}^{2+}$ was electrospun by means of a labscale device[12] (Figure 1). The electrospinning was carried out with a syringe connected to a syringe pump and a stainlesssteel needle $(0.7 \mathrm{~mm})$. A high voltage power of $30 \mathrm{kV}$ was applied between the needle and the grounded collector. The latter was placed at $10-20 \mathrm{~cm}$ from the needle. The flow rate of the solution was adjusted at $0.1 \mathrm{~mL} / \mathrm{h}$.

\subsubsection{Growth of MOF-5 crystals on the electrospun PVA $\mathrm{Zn}^{2+}$ nanofiber surface}

$12.5 \mathrm{mg}$ of 1 , 4-benzene dicarboxylic acid $\left(\mathrm{H}_{2} \mathrm{BDC}\right)$ were dissolved in $1 \mathrm{~mL}$ of methanol with $20 \mu \mathrm{L}$ of TEA under a gentle stirring for few minutes. A portion of the obtained PVA $/ \mathrm{Zn}^{2+}$ nanofibers was then immersed into the previous solution for $30 \mathrm{~s}, 1 \mathrm{~min}$ and $2 \mathrm{~min}$. Then, the nanofibers were washed two times with fresh methanol. The fiber modification process is depicted in Figure 2.

\subsection{Characterization}

\subsubsection{Scanning electron microscopy (SEM)}

The morphological studies of different nanofibers were carried out using scanning electron microscopy (HITACHI S-2360N) (Laboratoire de Physique et Mécanique Textiles (LPMT)). Prior to the analysis, all samples were metallized in order to render

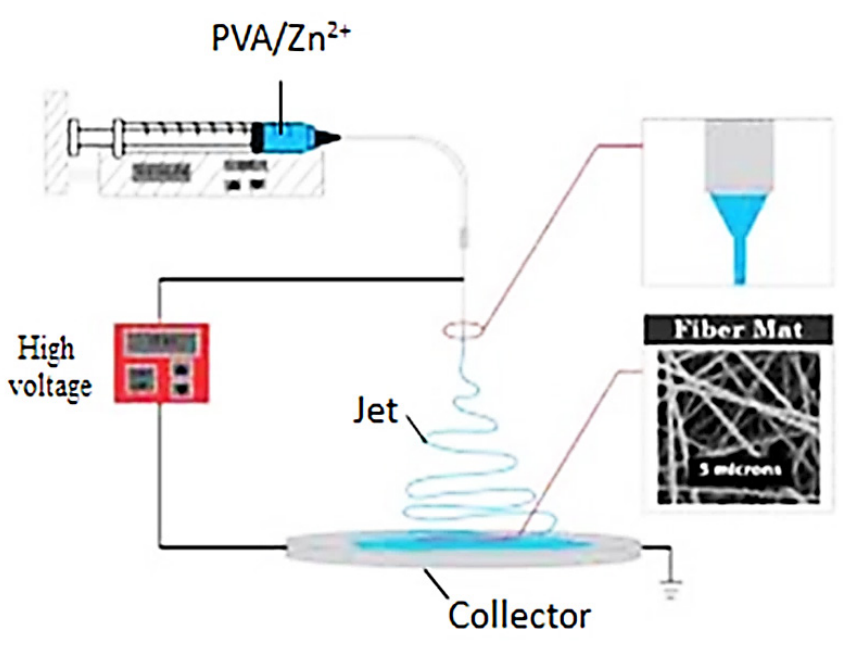

Figure 1. Schematic illustration of electrospinning set up.

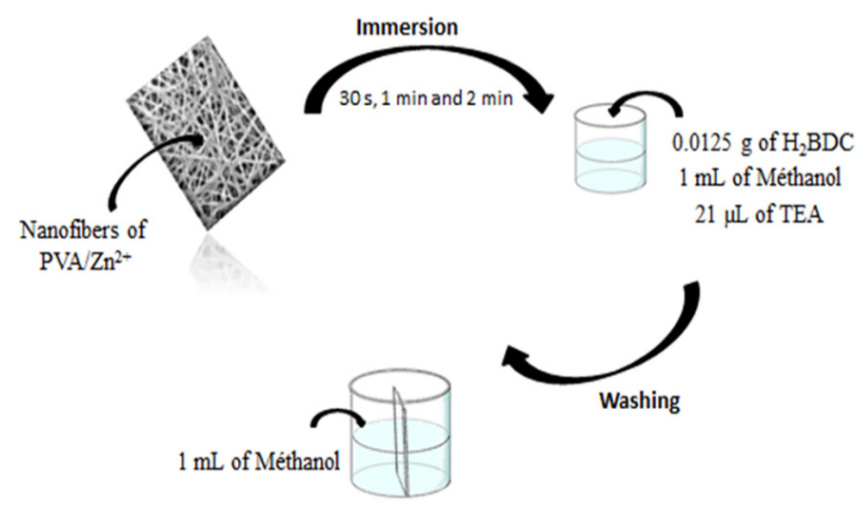

Figure 2. Schematic illustration of in situ growth of MOF-5 on $\mathrm{PVA} / \mathrm{Zn}^{2+}$ surface nanofibers. 
their surface conductive for SEM purposes.

After studying the morphology of nanofibers using SEM, 30 individual nanofibers were taken to calculate the average diameter using processing and image analysis software (Image J).

\subsubsection{Powder $X$-ray diffraction}

XRD diffractograms were obtained by a PANalytical X'Pert PRO MPD diffractometer using $\mathrm{Cu}-\mathrm{K} \alpha$ radiation $(\lambda=$ $1.542 \AA$ ) from 5 to $80^{\circ}$ for investigating the crystallization behavior.

\subsubsection{Fourier transform infrared spectroscopy}

The structural analysis of the synthesized fibers was analyzed by the attenuated total reflectance Fourier transform infrared (ATR-FTIR) spectra and was collected using Perkin Elmer Spectrum, over the wave number range between 4000 and $400 \mathrm{~cm}^{-1}$.
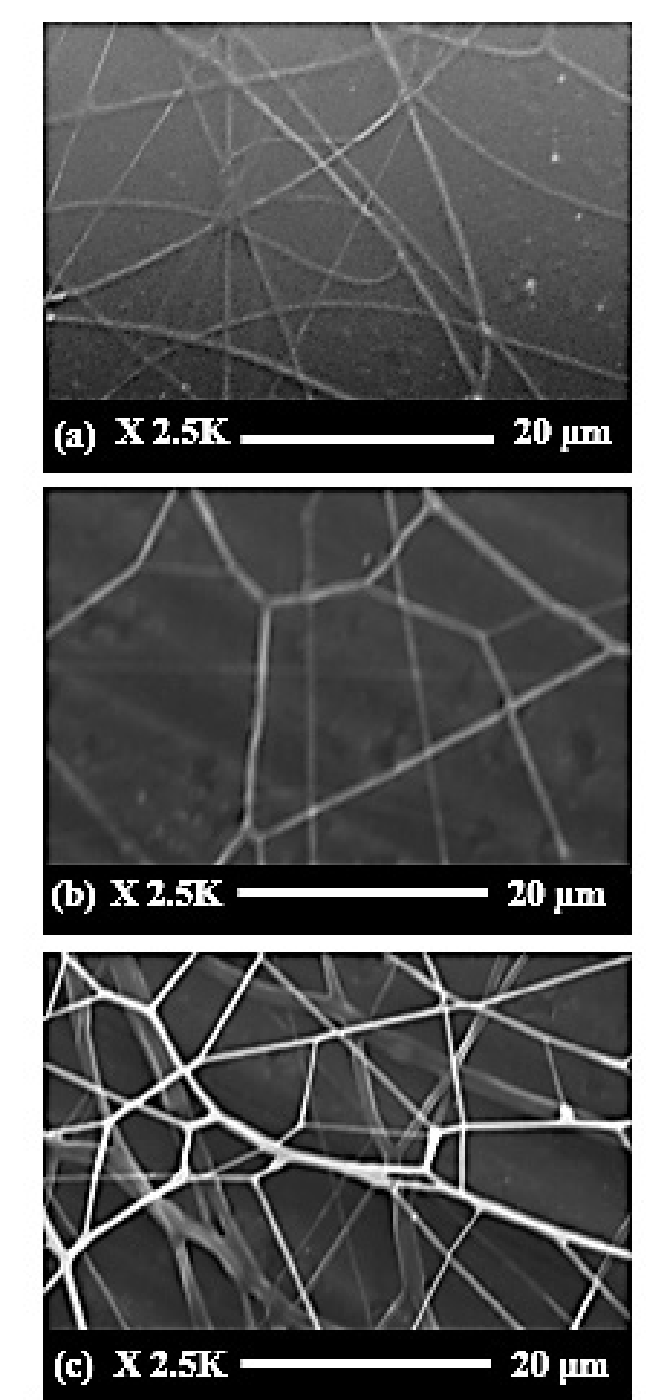

\section{Results and discussion}

\subsection{Characterization of growth of MOF-5 by scanning electron microscopy micrographs}

\subsubsection{Effect of the Tip-collector distance on the electrospinning of PVA/Zn ${ }^{2+}$}

As already pointed out in the literature,[24] the distance between the tip of the needle and the collector is an important factor for the electrospinning process and has a strong effect on the morphology of fibers.

In order to optimize this parameter, we investigated the effect of working distance on the PVA/ $\mathrm{Zn}^{2+}$ solution electrospinning. The spinning conditions mentioned below were adjusted; thus, the voltage was fixed at $30 \mathrm{kV}$, the concentration at $10 \mathrm{wt} \%$, the flow rate at $0.1 \mathrm{~mL} / \mathrm{h}$ and the distance was changed from 10 to $15 \mathrm{~cm}$. Effect of tip-target distance on fiber morphology is shown on Figure 3.

From Figure 3, we concluded that the diameter of fiber became enormously larger as the tip to collector distance increased.
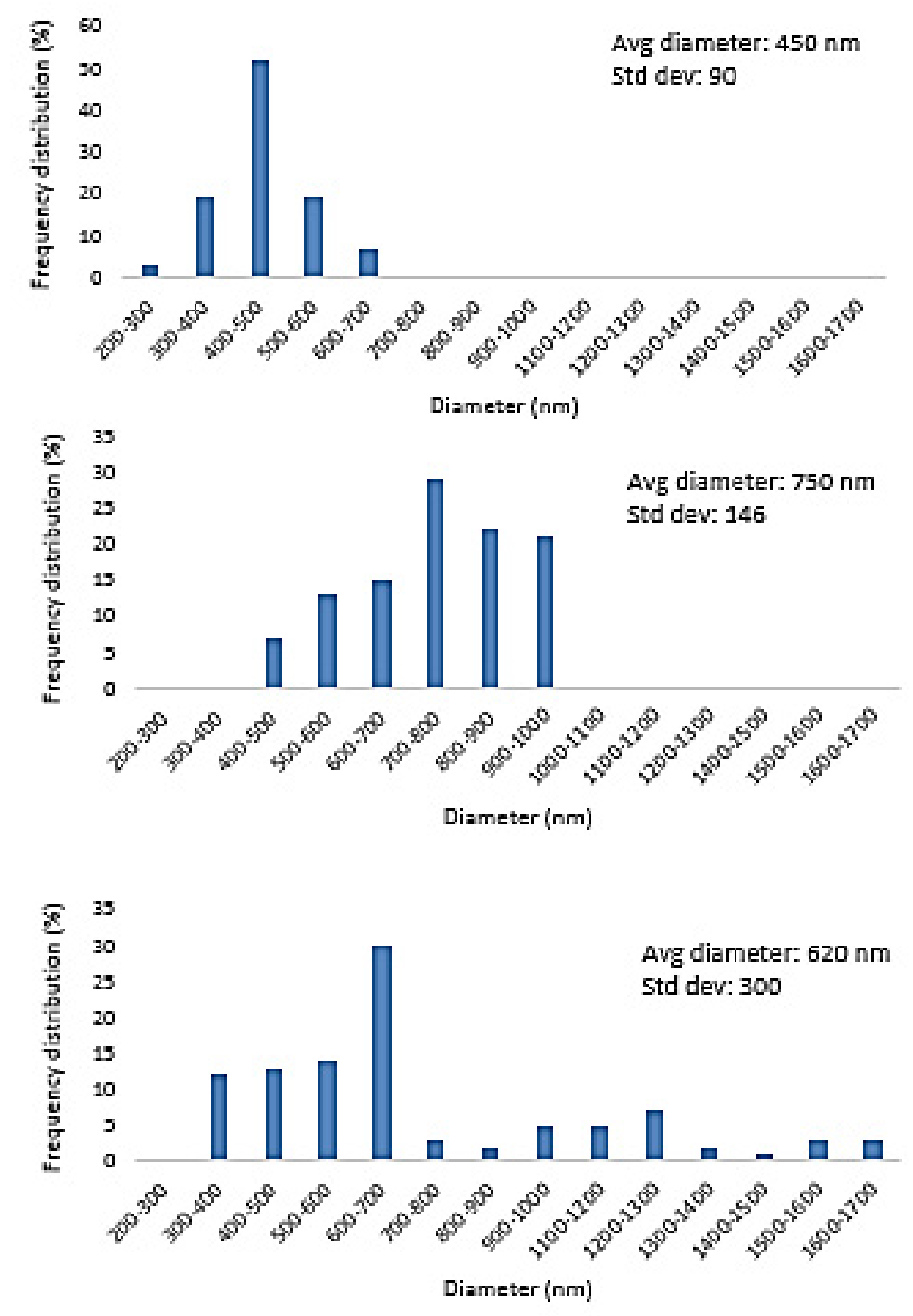

Figure 3. Effect of tip-target distance on fiber morphology from $10 \mathrm{wt} \% \mathrm{PVA} / \mathrm{Zn}^{2+}$ solution (voltage: $30 \mathrm{kV}$, flow rate: $0.1 \mathrm{~mL} / \mathrm{h}$ ). Tip-target distance: (a) $10 \mathrm{~cm}$; (b) $13 \mathrm{~cm}$ and (c) $15 \mathrm{~cm}$. Original magnification $2.5 \mathrm{k}$. 

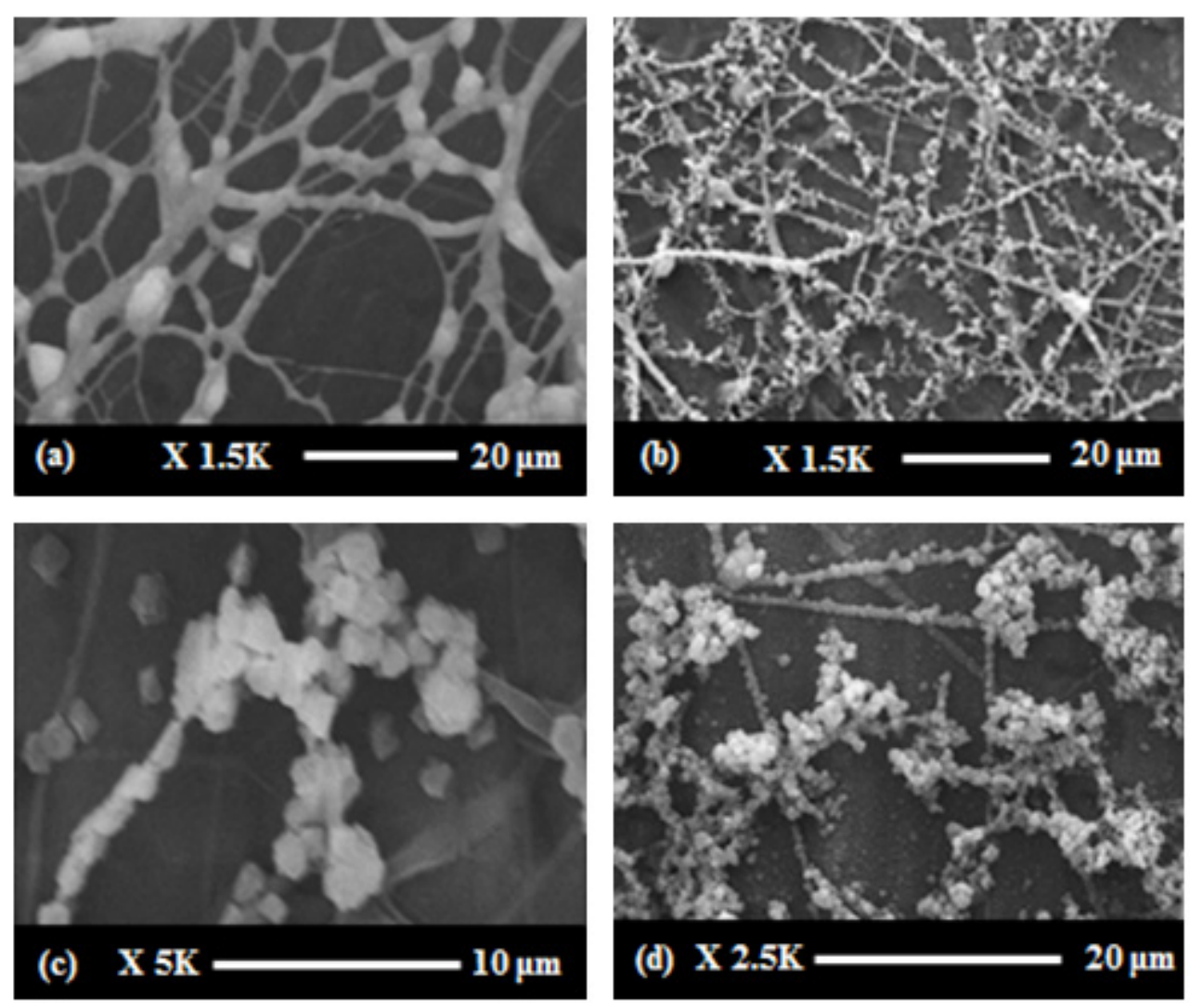

Figure 4. Effect of immersion time on fiber morphology from $10 \mathrm{wt} \% \mathrm{PVA} / \mathrm{Zn}^{2+}$ solution (voltage: $30 \mathrm{kV}$, flow rate: $0.1 \mathrm{~mL} / \mathrm{h}$ and tip-target distance: $10 \mathrm{~cm}$ ), (a) after immersion for $30 \mathrm{~s}$ (b and c) after immersion for $1 \mathrm{~min}$ and (d) after immersion for 2 min. Originals magnifications $1.5 \mathrm{k}, 2.5 \mathrm{k}$ and $5 \mathrm{k}$.

In fact, the diameter size varied from $450 \mathrm{~nm}$ to $750 \mathrm{~nm}$ in the range of 10 to $13 \mathrm{~cm}$, respectively. But a working distance of $15 \mathrm{~cm}$ led to the production of nanofibers with an average diameter of $620 \mathrm{~nm}$.

At different distances, the average diameter was observed to increase from 450 to $620 \mathrm{~nm}$ with increasing the distance. This indicates that the morphology, diameter size, and uniformity of the electrospun PVA/Zn ${ }^{2+}$ nanofibers are significantly influenced by the distance.

The best and uniform morphology of electrospun nanofibers was obtained when we fixed the distance spinning at $10 \mathrm{~cm}$. In this case, the average diameter was below $500 \mathrm{~nm}$.

\section{1. 2. Crystal growth of MOF-5 in PVA/Zn ${ }^{2+}$ nanofibers}

The growth of MOF-5 on the previously described nanofibers was tested by their immersion in a 1,4-benzene dicarboxylate (BDC) solution. In fact, MOF-5 is the first robust MOF primarily prepared by Yaghi et al. in 1999 [25]. This material has a facecentered cubic crystal structure with a cubic basic unit. The corresponding structure is formed by $\left[\mathrm{Zn}_{4} \mathrm{O}_{6}{ }^{+}\right.$metal cluster as corner and linked by 1,4-benzene dicarboxylate (BDC) as edge. A large empty cavity is formed for each unit cell and until $61 \%$ of the crystal space is accessible to guest species [25].

SEM images (Figure 4) revealed that the MOF-5 nanocrystals have grown along the $\mathrm{PVA} / \mathrm{Zn}^{2+}$ nanofibers that served as crystal growth template by providing the $\mathrm{Zn}^{2+}$ ions. SEM images were recorded for different immersion times as depicted in Figure $4 \mathrm{a}$ and $4 \mathrm{~b}$. They evidenced that the nucleation of MOF5 is very fast and that crystal seeds were formed after $30 \mathrm{~s}$. As expected, increasing the immersion time enhanced the coating of nanofibers surface by crystals. The best homogeneous, compact and continuous growth of MOF- 5 was obtained after 1 min immersion time. Continuous and compact hierarchical structures were observed.

By lengthening the deposition time to $2 \mathrm{~min}$, the metallic cations were released outside fibers leading to the formation of MOF-5 aggregates (Figure 4d).

\section{2. Characterization of growth of MOF-5 by Fourier transform infra-red (FT-IR) spectroscopy}

Fourier transform infrared (FTIR) spectra were recorded for MOF-5, PVA and for the MOF-5-modified nanofibers, as shown in Figure 5.

The spectrum of the modified nanofibers (Fig. 5c) confirmed the growth of MOF- 5 crystal on the PVA. Actually, it exhibited two characteristic adsorptions bands corresponding to the symmetric and asymmetric vibrations of BDC bands at 1575 and $1391 \mathrm{~cm}^{-1}$, respectively. These values are consistent with the presence of $\mathrm{CO}_{2}^{-}$groups coordinating to zinc. Moreover, the vibrational bands in the range of $730-850 \mathrm{~cm}^{-1}$ were assigned to the out-of-plane vibrations of terephthalates. Finally, the absorption peak at $530 \mathrm{~cm}^{-1}$ is relevant to the secondary building unit (SBU) of MOF-5, corresponding to 


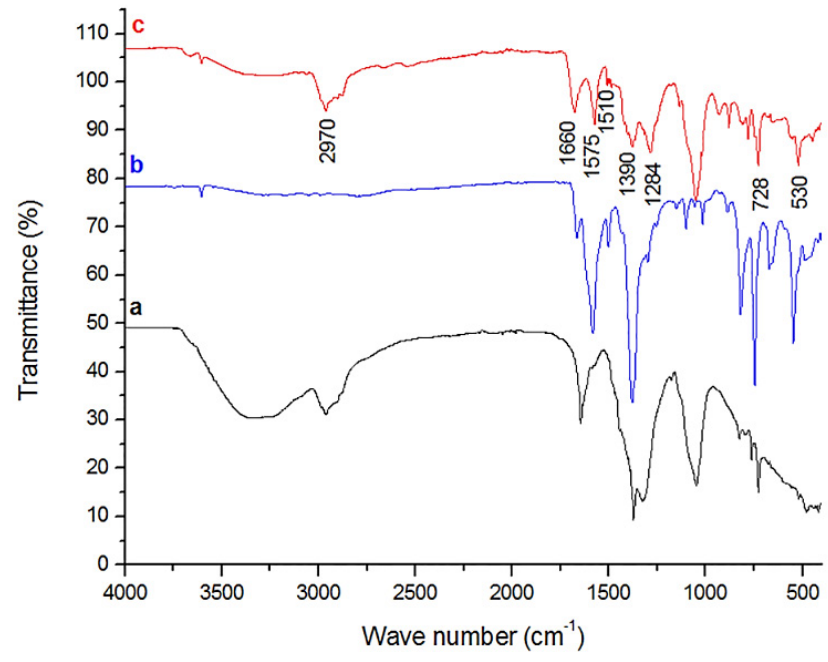

Figure 5. Vibrational spectra of MOF-5, a) pure PVA, b) bulk reference of MOF-5, c) MOF-5 grown on the fiber surface of PVA/Zn ${ }^{2+}$ via immersion process.

the $(\mathrm{Zn}-\mathrm{O})$ vibration of tetrahedral coordinated $\mathrm{Zn}_{4} \mathrm{O}$ cluster [26].

It is worthy to note that the spectra revealed the presence of the characteristic bands of PVA. Indeed, the observed bands at 2850-3050 $\mathrm{cm}^{-1}, 1100 \mathrm{~cm}^{-1}$ and $1660 \mathrm{~cm}^{-1}$, were attributed to the $\mathrm{C}-\mathrm{H}$ broad alkyl stretching band, the $\mathrm{C}-\mathrm{O}$ stretching and carbonyl groups of pure PVA. However, the typical vibrations of hydrogen bonded hydroxyl groups (3200-3570 $\mathrm{cm}^{-1}$ ) were dramatically attenuated in the modified-fibers spectrum indicating the strong interactions between hydroxyl groups and MOF's crystals.

\section{3. Characterization of growth of MOF-5 by powder X-ray diffraction analysis}

The XRD patterns of MOF-5 material and MOF- 5 growth into the PVA $/ \mathrm{Zn}^{2+}$ nanofibers are shown in Figure 6. The MOF-5 nanocrystals grown on the PVA $/ \mathrm{Zn}^{2+}$ surface nanofibers showed a diffraction similar to that of bulk MOF-5 used as reference [25]. The obtained diffractogram illustrated that MOF-5 was well crystallized on the nanofibers surface according to a cubic

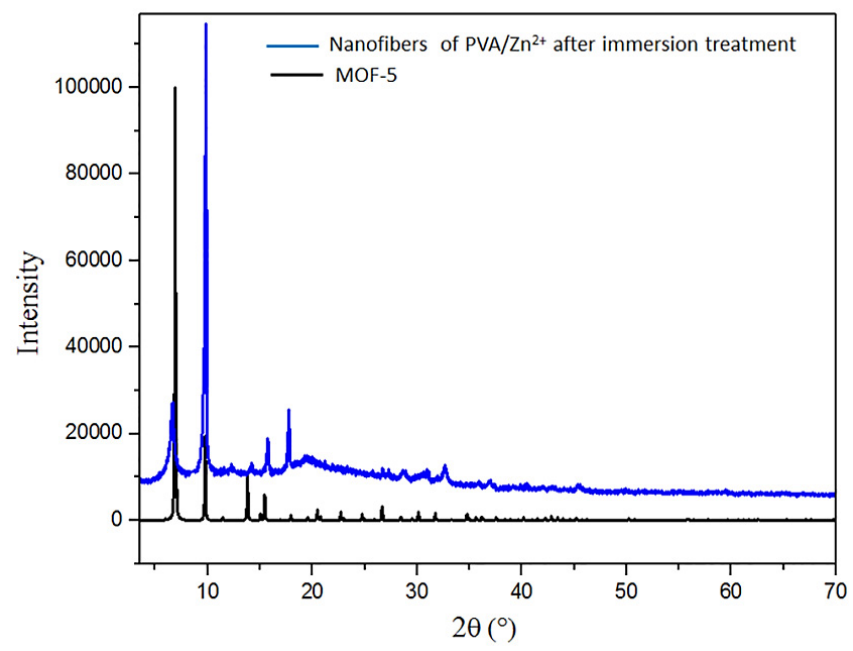

Figure 6. X-ray diffraction patterns of the bulk reference and the MOF5 growth into the PVA/Zn ${ }^{2+}$ nanofibers. symmetry with a space group Fm-3m and a $25.8849 \AA$. Note that the diffraction peak at $2 \theta=19.4^{\circ}$ was attributed to the neat PVA [27].

\section{Conclusions}

In this research, we have described a novel approach to produce MOF- 5 hierarchical structures. This approach involves the use of electrospinning for the preparation of nanofibers charged with metallic cation. Accordingly, $\mathrm{Zn}^{2+}$ loaded PVA nanofibers have been electrospun. These nanofibers were used as template structure for the MOF- 5 growth. In fact, when immersed in a linker solution, they acted as a metal source for the surrounding medium. The released $\mathrm{Zn}^{2+}$ assembled rapidly with the linker and thereby led to the growth of MOF- 5 crystal on the surface of nanofibers. Both morphology and conversion yield were found to be hardly affected by the immersion time in the organic linker solution. The best result was obtained after a 1 minute-immersion leading to a compact fibers coating. For longer duration, MOF-5 aggregates were obtained outside the fibers. The process allows an easy tuning of the composite structures. The described method is highly versatile and allows access to a wide range of MOF's hierarchical structures with different morphology, size and composition. The obtained results provide useful information to elaborate non-woven functional textile, charged with different MOFs, for various applications in different areas such as sensors, photo catalytic systems, electrodes and membranes.

\section{ACKNOWLEDGEMENTS}

This research was supported by the Ministry of Higher Education and Scientific Research, Tunisia.

\section{References}

[1] Zagho, M.M. and Elzatahry, A. (2016). Recent Trends in Electrospinning of Polymer Nanofibers and their Applications as Templates for Metal Oxide Nanofibers Preparation. In Electrospinning - Material, Techniques, and Biomedical Applications Edited by Haider, S. and Haider, A. InTech press.

[2] Brown, P. J. and Stevens, K. (2007). Nanofibers and nanotechnology in textiles. CRC Press (Boca Raton Boston New York Washington, DC) and WOODHEAD PUBLISHING LIMITED (Cambridge, England).

[3] Wendorff, J.H., Agarwal, S. and Greiner, A. (2012). Electrospinning: Materials, Processing, and Applications. Wiley-VCH Verlag \& Co. KGaA (Weinheim, Germany).

[4] Li, D., and Xia, Y. (2004). "Electrospinning of nanofibers: reinventing the wheel?”. Advanced materials, 16 (14), 1151-1170.

[5] Reneker, D. H., and Yarin, A. L. (2008). Electrospinning jets and polymer nanofibers. Polymer, 49 (10), 2387-2425.

[6] Jia, Y. T., Gong, J., Gu, X. H., Kim, H. Y., Dong, J., and Shen, X. Y. (2007). Fabrication and characterization of poly (vinyl alcohol)/chitosan blend nanofibers produced by electrospinning method. Carbohydrate Polymers, 67 (3), 403-409. 
[7] Wang, X., Chen, X., Yoon, K., Fang, D., Hsiao, B. S., and Chu, B. (2005). High flux filtration medium based on nanofibrous substrate with hydrophilic nanocomposite coating. Environmental science \& technology, 39 (19), 7684-7691.

[8] Shan, D., Qian, B., Ding, S. N., Zhu, W., Cosnier, S., and Xue, H. G. (2010). Enhanced solid-state electrochemiluminescence of tris (2, 2'-bipyridyl) ruthenium (II) incorporated into electrospun nanofibrous mat. Analytical chemistry, 82 (13), 5892-5896.

[9] Greiner, A., and Wendorff, J. H. (2007). Electrospinning: a fascinating method for the preparation of ultrathin fibers. Angewandte Chemie International Edition, 46 (30), 56705703.

[10] Zheng, J., Zhang, H., Zhao, Z., and Han, C. C. (2012). Construction of hierarchical structures by electrospinning or electrospraying. Polymer, 53 (2), 546-554.

[11] Zander, N. E. (2013). Hierarchically structured electrospun fibers. Polymers, 5 (1), 19-44.

[12] Khenoussi, N., Drean, E., Schacher, L., Adolphe, D. and Balard, H. (2012). Nanofibers production - Study and development of electro-spinning device. Experimental Techniques, 36 (2), 32-39.

[13] Rose, M., Böhringer, B., Jolly M., Fischer, R., Kaskel, S. (2010). MOF Processing by Electrospinning for Functional Textiles. Advanced Engineering Materials, 13 (4), 356-360.

[14] Ostermann, R., Cravillon, J., Weidmann, C., Wiebcke, M., and Smarsly, B. M. (2011). Metal-organic framework nanofibers via electrospinning. Chemical Communications, 47 (1), $442-444$.

[15] Ren, J., Musyoka, N. M., Annamalai, P., Langmi, H. W., North, B. C., and Mathe, M. (2015). Electrospun MOF nanofibers as hydrogen storage media. international journal of hydrogen energy, 40 (30), 9382-9387.

[16] Jin, R., Bian, Z., Li, J., Ding, M., Gao, L. (2013). ZIF-8 crystal coatings on a polyimide substrate and their catalytic behaviours for the Knoevenagel. Dalton Transactions, 42 (11), 3936-3940.

[17] Lian, Z., Huimin, L., Zhaofei, O. (2014). In situ crystal growth of zeolitic imidazolate frameworks (ZIF) on electrospun polyurethane nanofibers. Dalton Transactions, 43 (18), 6684-6688.
[18] Laurila, E., Thunberg, J., P. Argent, S., R. Champness, N., Zacharias, S., Westman, G, Öhrström, L. (2015). Enhanced Synthesis of Metal-Organic Frameworks on the Surface of Electrospun Cellulose Nanofibers. Advanced Engineering Materials, 17 (9), 1282-1286.

[19] Kaskel, S. (2016).The Chemistry of Metal-Organic Frameworks Synthesis, Characterization and Applications. Wiley-VCH Verlag GmbH \& Co. Weinheim, (Germany).

[20] Bradshaw, D., Garai, A. and Huo, J. (2012). Metal-organic framework growth at functional interfaces: thin films and composites for diverse applications. Chemical Society Reviews, 41 (6), 2344-2381.

[21] Furukawa, S., Reboul, J., Diring, S., Sumida, K. and Kitagawa, S. (2014). Structuring of metal-organic frameworks at the mesoscopic/macroscopic scale. Chemical Society Reviews, 43 (16), 5700-5734.

[22] Falcaro, P., Buso, D., Hill, A.J. and Doherty, C.M. (2012). Patterning Techniques for Metal Organic Frameworks. Advanced Materials, 24 (24), 3153-3168.

[23] Yeo, Z.Y., Chai, S-P., Zhu, P.W. and Mohamed, A.R. (2014). An overview: synthesis of thin films/membranes of metal organic frameworks and its gas separation performances. RSC Advances, 4 (97), 54322-54334.

[24] Atabey, E., Wei, S., Zhang, X., Gu, H., Yan, X., Huang, Y., Shao, L., He, Q., Zhu, J., Sun, Luyi., Kucknoor, A.S., Wang, A. and Guo, Z.S. (2013). Fluorescent electrospun polyvinyl alcohol/CdSe@ZnS nanocomposite fibers. Journal of Composite Materials, 47 (25), 3175-3185.

[25] Li, H., Eddaoudi, M., O'Keeffe, M., and Yaghi, O. M. (1999). Design and synthesis of an exceptionally stable and highly porous metal-organic framework. Nature, 402 (6759), 276279.

[26] Sabouni, R., Kazemian, H., and Rohani, S. (2010). A novel combined manufacturing technique for rapid production of IRMOF-1 using ultrasound and microwave energies. Chemical Engineering Journal, 165 (3), 966-973.

[27] Assender, H. E., and Windle, A. H. (1998). Crystallinity in poly (vinyl alcohol). 1. An X-ray diffraction study of atactic PVOH. Polymer, 39 (18), 4295-4302. 